\title{
MONS: Measuring Oscillations in Nearby Stars
}

\author{
H. Kjeldsen ${ }^{1}$, T. R. Bedding ${ }^{2}$, J. Christensen-Dalsgaard ${ }^{1}$
}

Abstract. MONS is a proposed space mission to study stellar oscilla-
tions. We briefly describe the main features of the mission - more details
are available at the MONS Home Page (http://www obs aau.dk/MONS).

A proposal for MONS (Measuring Oscillations in Nearby Stars) was submitted to the Danish Small Satellite Programme in March 1999 and is currently being evaluated. The purpose of MONS is to strengthen substantially the fundamental basis of astrophysics which stellar astrophysics provides. This will be accomplished by carrying out observation of stellar oscillations at a greatly improved level of sensitivity, to allow detailed investigations of solar-like oscillations in a sample of nearby stars. Despite intensive efforts, such oscillations have proven impossible to study from the ground because of the effects of the Earth's atmosphere, whereas observation from space with a modest telescope is relatively straightforward.

MONS will measure oscillations in about 25 nearby stars by monitoring the brightness and colour fluctuations in each star for about one month. The primary aim is to provide new insights into the structure and evolution of stars, using them as laboratories to understand physics under extreme conditions. Secondary mission objectives are (i) to study the structure and evolution of stars hotter and more massive than the Sun ( $\delta$ Scuti and rapidly oscillating Ap stars) by measuring their oscillations; and (ii) to study variability in a large sample of stars of all types.

The telescope is a $34-\mathrm{cm}$ reflector, with a dichroic beamsplitter to form side-by-side blue and red stellar images. A broad range of possible targets and minimal interruptions in the observations will be ensured by choosing an orbit with a high apogee and inclination. We will obtain parallel scientific information from two Star Trackers, whose primary purpose is to sense the spacecraft attitude. These will provide long-term monitoring of around 1000 stars of all types, with a photometric precision substantially exceeding what is available from ground-based observations.

\footnotetext{
${ }^{1}$ Theoretical Astrophysics Center, Danish National Science Foundation, Institute of Physics and Astronomy, Aarhus University, DK-8000 Aarhus C, Denmark

${ }^{2}$ School of Physics, University of Sydney 2006, Australia
} 